\title{
Research on Vectorization Method of Architectural Floor Plan Images
}

\author{
Rong $\mathrm{Li}^{1}$, Kun Zou ${ }^{1,2, ~}$,Wensheng Li ${ }^{1,2}$, Liang Yang ${ }^{1}$ \\ ${ }^{1}$ University of Electronic Science and Technology of China, Zhongshan Institute, Zhongshan, 528402, China \\ ${ }^{2}$ School of Computer Science andEngineering, University of Electronic Science and Technology of China, \\ Chengdu, 611731, China \\ * Corresponding Author: Kun Zou
}

Keywords: Morphological, Threshold segmentation, Contour detection, Vector, Hough transform

\begin{abstract}
A method of automatic vectorization is proposed for nonstandard architectural floor plan images. It contains four steps: image preprocessing, segmentation, vector, classification. Use different algorithms in different regions. Classify the lines after vectorization according to geometric characteristics and prior knowledge. The result of multiple experiments has testified the feasibility and efficiency of the algorism. The method can be used in furniture customization, home decoration display, virtual reality and other application fields.
\end{abstract}

\section{Summary}

With the development of computer science, graph recognition technology has been widely used in many types of technical documents and drawings analysis. But the research on automatic analysis and processing of construction drawing is still relatively few.

Although the scientists at home and abroad continue to improve the vectorization of raster images and vector method, the existing vectorization software level is not very high, mainly reflected in the recognition processing speed, anti-noise, various characters and unsatisfying curve. For architectural drawings, most of the objects in the current system are the standard architectural design, not the floor plans after prettification in the sale of the housings.

Aiming at the demands of furniture customization, home display and other application areas, this paper achieve rapid automation, vectorization and classification based on the common housing units map. The image requirements are relatively low and the architectural design is unprofessional. The input can be the floor plan images in the publicity materials when people purchase the housing.

\section{Overall Scheme}

The early architectural floor images are generally drawn on paper, which can be transformed into images by digital sweeping instruments. At present, floor images can be easily obtained from related channels. Images are the most available and accessible to ordinary people. According to the vectorization of these images, the results can be imported into the 3D modeling software to generate three-dimensional apartment drawings, which can be convenient for home decoration design or furniture customization.

\section{Image Pretreatment}

The following tasks are required to fulfill in the image preprocessing stage, such as size constraint, image enhancement, noise removal and so on.

First, the image can be partly cutting. This process requires manual intervention. But, due to the simple operation and quick speed of the cutting, it does not increase the difficulty of operation. We can quickly remove a lot of non-building component content, such as real name, advertising words and so on. 
Then, the size constraints should be taken. Different image sources often lead to different image size. To deal with the later segmentation and recognition, the sizes should be unified first.

Finally, image synthesis is done to achieve the goal of enhancement.

After doing the above treatment, we use the appropriate size of the kernel to do morphological operations to remove some noise.

\section{Image Segmentation and Vectorization}

Obtainment of External Overall Outline. In addition to the relevant element information, the apartment layout map contains some other content. Especially, the ordinary home buyers are prone to access the apartment layout diagrams processed and beatified by the advertising company in the late stage. Therefore, we will focus the apartment layout area and external contour to acquire the entire overall outline in the first step.

In terms of the gray image, we should conduct the binarization and contour detection after pretreatment. The external contour integral, the contour information windlow and the door are generally contained in this profile.

In terms of the color images, there is usually a color or texture in addition to the type information. To obtain the overall contour of the apartment, the first step is to partition the background and the apartment, and then use the region growing algorithm. The color values of pixels in fixed range satisfy the following conditions:

$\operatorname{src}($ seed.x,seed.y)r-lo_diffr $<=\operatorname{src}(x, y) r<=\operatorname{src}($ seed.x,seed.y)r+up_diffr and

$\operatorname{src}($ seed.x,seed.y)g-lo_diffg $<=\operatorname{src}(\mathrm{x}, \mathrm{y}) \mathrm{g}<=$ src(seed.x,seed.y)g + up_diffg and

$\operatorname{src}(\operatorname{seed} . x$, seed.y)b-lo_diffb $<=\operatorname{src}(x, y) b<=\operatorname{src}(\operatorname{seed} . x$, seed.y) $b+$ up_diffb.

Wall Inspection. According to the shape, the apartment layout drawings can be divided into two categories: fine structure and block structure. The former category contains a variety of thin, hollow circular arc, and text object; the latter category includes a variety of specific symbols such as arrows, solid and solid circle. If the width of the object in the image is greater than a certain value, the object belongs to the area, otherwise it is a thin wire structure. As one of the most important components of the apartment layout, the segmentation and detection result of the wall directly related to the final 3D reconstruction effect is correct. The wall shape is thick line entities in some image, belonging to the area. Some of the images are rough, some images of the wall are drawn completely in thin lines. Different wall forms are suitable to different detection methods.

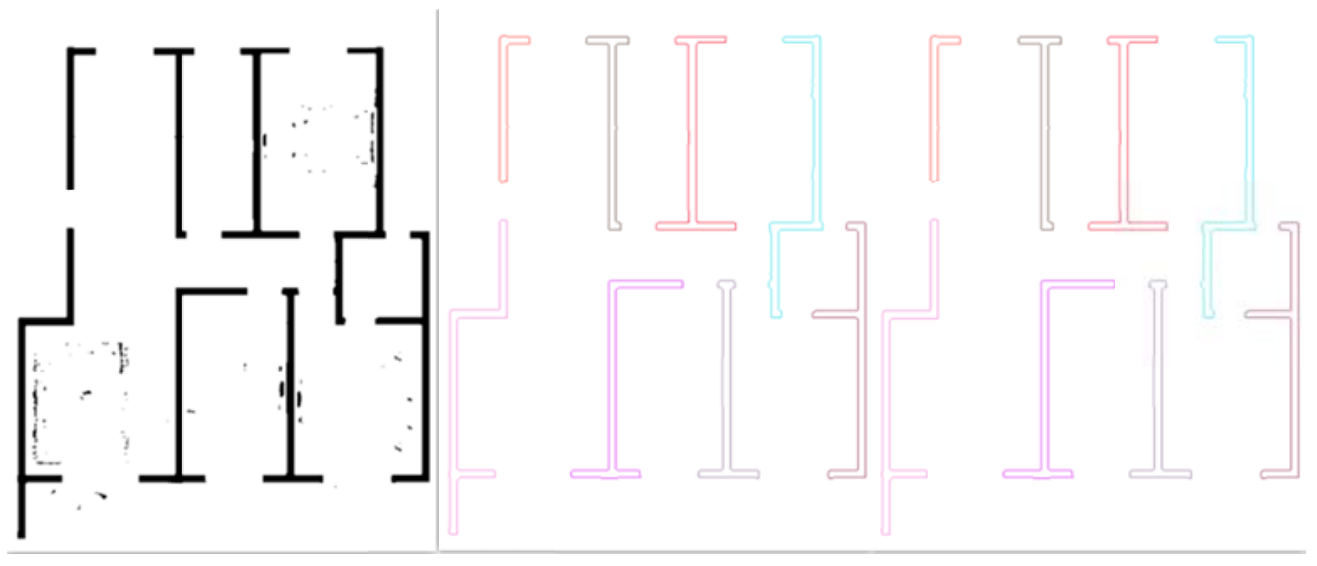

Figure 1. Threshold segmentation Figure 2. Outline detection Figure 3. Outline filtering 


$\begin{array}{lllllllllllllllllllll}1 & 1 & 1 & 1 & 1 & 1 & 1 & 1 & 1 & 1 & 1 & 0 & 0 & 0 & 0 & 0 & 0 & 0 & 0 & 1 \\ 1 & 0 & 0 & 0 & 0 & 0 & 0 & 0 & 0 & 1 & 1 & 0 & 0 & 0 & 0 & & 0 & 0 & 0 & 0 & 1 \\ 1 & 0 & 0 & 0 & 0 & 0 & 0 & 0 & 0 & 1 & 1 & 0 & 0 & 0 & 0 & 0 & 0 & 0 & 0 & 1 \\ 1 & 0 & 0 & 0 & 0 & 0 & 0 & 0 & 0 & 1 & 1 & 0 & 0 & 0 & 0 & 0 & 0 & 0 & 0 & 1 \\ 1 & 0 & 0 & 0 & 0 & 0 & 0 & 0 & 0 & 1 & 1 & 1 & 1 & 1 & 1 & & 1 & 1 & 1 & 1 & 1\end{array}$

Figure 4. Convolution kernels

The vectorization process of the composite wall is shown in Figure 5, Figure 6 and Figure 7. Figure 5 shows the original floor plan; Figure 6 shows the thick lines part of the floor plan; Figure 7 shows the thin lines part of the floor plan.

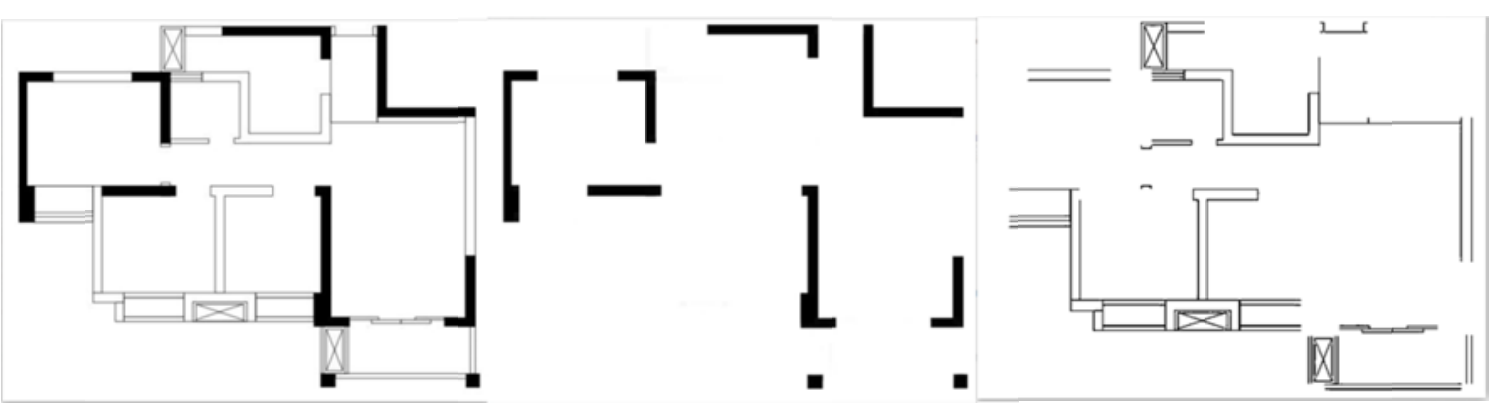

Figure 5. original floor plan Figure 6. Thick lines part Figure 7. Thin lines part

The vectorization of architectural floor plan drawn by the thin lines is shown inf Figure 8.

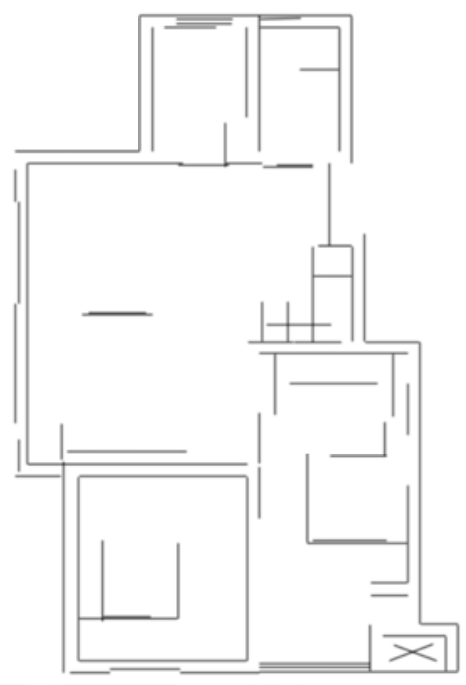

Figure 8. Line segment detection

Inspections of the Door, the Window and the Balcony.Based on the detection of the wall line and the outer contour line, the features of the exterior contours of the window, balcony and door are used to find out the location of the balcony, window and entrance door with the differences between the inside and outside contours. The specific process is shown in Figure 9, Figure 10 and Figure 11. The outline of the wall and external contours are drawn into the same image in Figure 9. The outline of the wall is enlarged to avoid the possibility of a little deviation in the edge position during the detection of the outer contour in Figure 9. We subtract the wall bold drawing from Figure 10 to get Figure 10. We examine the contours of Figure 10, and then classify the resulting contour sets to obtain the categories of doors, balconies and windows. The basis for judging the balcony is the longest perimeter. The basis of the discrimination gate is the number of the concentrated points of the contour. The rest are the outline of the window, and the result is shown in Figure 11. The red line indicates the wall; the green line represents the window; the blue line represents the balcony, and the yellow line indicates the entrance door. 


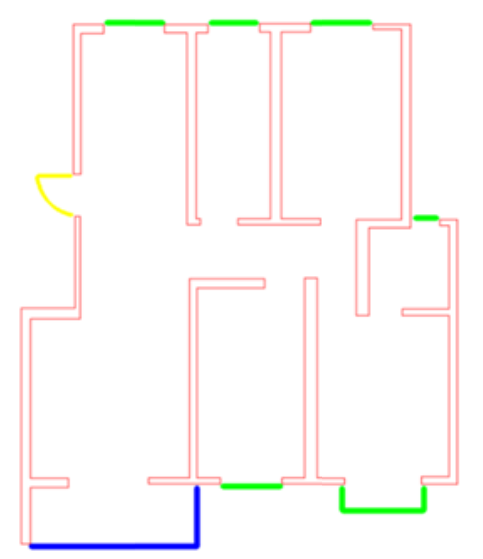

Figure 9. Combination of internal and external contours Figure

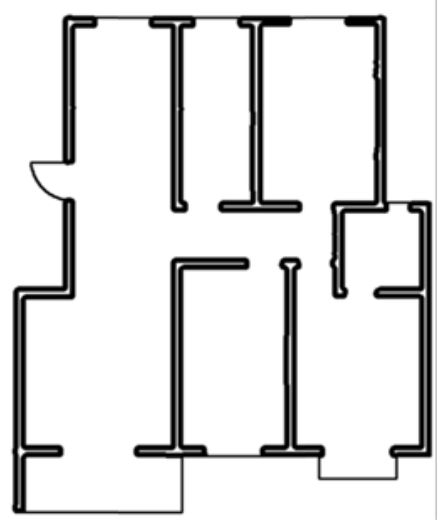

10. Difference between internal and external contours

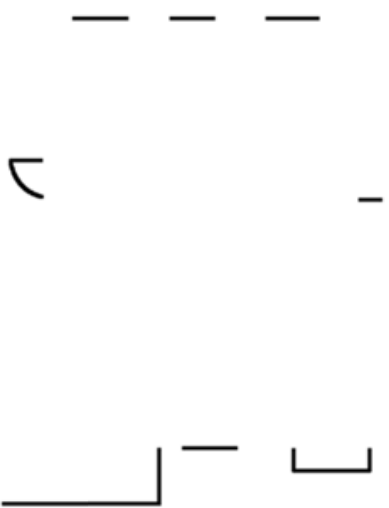

Figure 11. Detection result

\section{Conclusions}

We adopted the Visual Studio 2013 and opencv3.0 in the personal computer to realize the algorithm and teste the algorithm through many images of different shapes and different sources in the paper. In terms of the solid line walls, most of the images can be correctly segmented and vectorized and the walls, windows, balconies and internal doors can be identified, which is shown in Figure 13. We can also correctly identify and vectorized the floor plan drawn by solid-line and double-line without furniture. We save the coordinate information after vectorization, such as the XML file, and achieve the correct result in the later 3D reconstruction. 

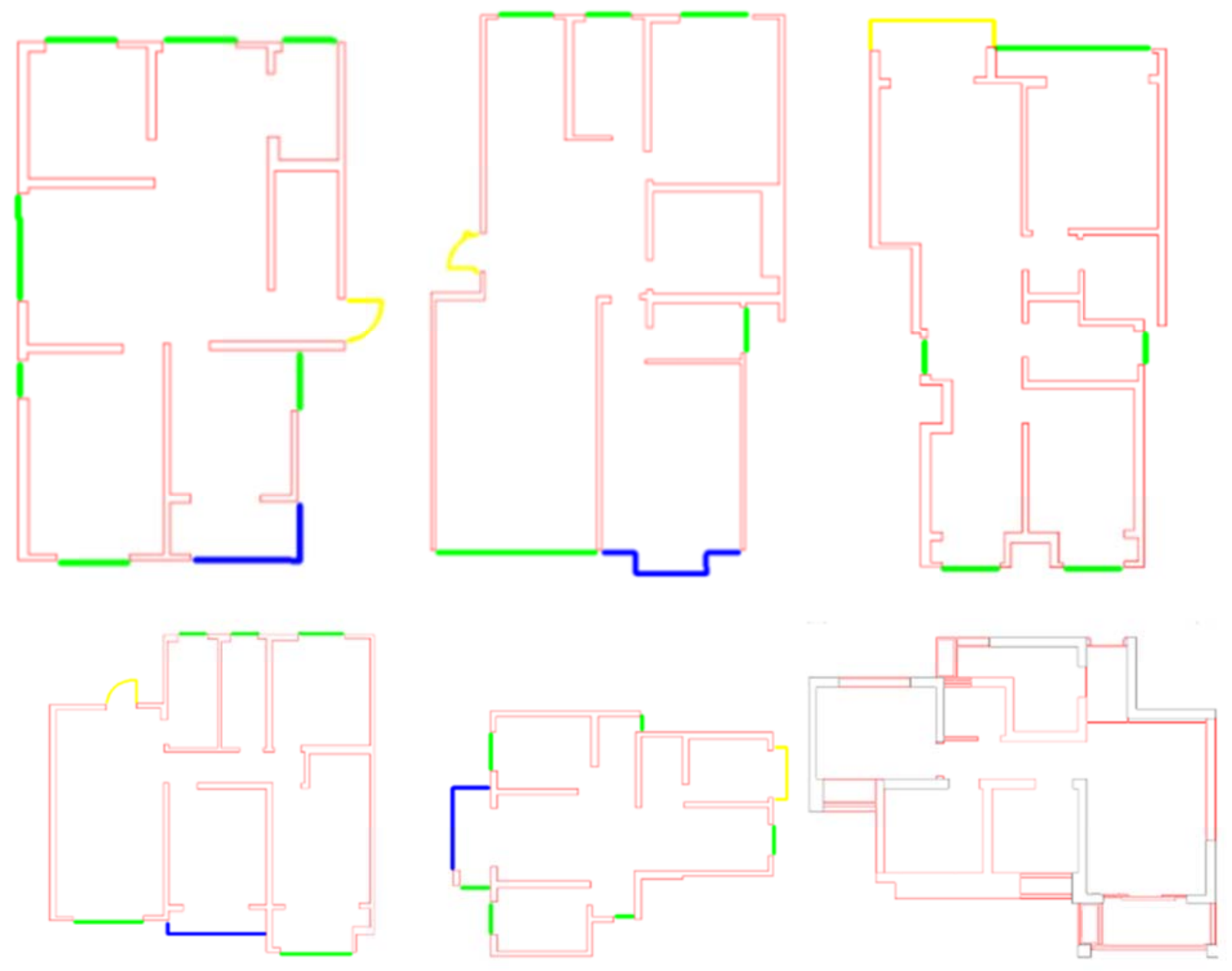

Figure 12. Some experimental results

The algorithm can realize the identification of household doors and windows in the balcony. The improvement of the algorithm to identify the internal door is the next step of the research. In addition, to improve the robustness of the algorithm to adapt to the apartment layout map with perspective and distortion and enhance the efficiency is the direction of our efforts.

Through the authentication of many images, we conclude that there is no fixed method of the vectorization for apartment layout drawing. We can consider all possibilities and allow the users to select the type of image recognition. We can also provide the choice of the style, such as the style of walls, doors and windows. We can achieve the different methods of vectorization according to different styles quickly and accurately to lay foundations for the 3D reconstruction of the next step.

\section{Acknowledgement}

This research was funded by National Natural Science Foundation (Grant No. 6150288). It was also the result of Cultivation Plan of Excellent Young Teachers of Universities and Colleges in Gugangdong Province (Grant No. YQ2015241) and Plan Project of Science and Technology of Zhongshan City (Grant No. 2017B1115).

\section{References}

[1] Zhou Jiliu, Guo Jing, Zhang Ye, Zhu Hui, Long Jianzhong, Chen Zhongling. A Study on Vectorization System of Engineering Drawings [J].Journal of Sichuan University (Natural Science Edition), 1998, 35(1): 65-70. 
[2] Ding Weidong, Yuan Jingqi. Study on Vectorization Algorithm of Engineering Drawing [J]. Computer Engineering, 2006, 32(24): 157-158+161.

[3] Lu Zongqi, Zhang Qiuping. Line Contour Tracking in EngineeringDrawing Vectorization [J]. Journal of Image a nd Graphics, 1997, 2(12): 878-882.

[4] Zhang Qing, Guo Wei. Depth images-based approach for 3D reconstruction [J].Journal of Huazhong University of Science and Technology (Natural Science Edition), 2008, 36(2): 88-91.

[5] Lu Guodong, Ruan Jianzhong, Jiang Tao, Peng Qunsheng. Research on 3D Reconstruction of Objects from Engineering Drawing [J].Journal of Computer Aided Design andComputer Graphics, 1999, 11(6): 516-520.

[6] Yin X, Wonka P, Razdan A. Generating 3D building models from architectural drawings: a survey [J]. IEEE Computer Graphics \& Applications, 2009, 29(1):20.

[7] Dori D, Liu W. Sparse Pixel Vectorization: An Algorithm and Its Performance Evaluation [J]. IEEE Transactions on Pattern Analysis \& Machine Intelligence, 1999, 21(3): 202-215.

[8] Lu T, Tai C L, Yang H, et al. A Novel Knowledge-Based System for Interpreting Complex Engineering Drawings: Theory, Representation, and Implementation [J]. IEEE Transactions on Pattern Analysis \& Machine Intelligence, 2009, 31(8):1444-1457. 